\title{
Antituberculosis treatment-induced hepatotoxicity: role of predictive factors
}

\author{
J Singh, A Arora, PK Garg, VS Thakur, JN Pande, RK Tandon
}

\begin{abstract}
Summary
Antituberculosis drug-induced hepatotoxicity is quite common. However, factors predicting its development are still controversial. The objective of the present study was to evaluate the role of certain factors (age and sex of the patient, alcoholism, chronic liver disease, hepatitis B virus carrier status, acetylator status, nutritional status and antituberculosis treatment (ATT) regimen) in predicting the development of ATT-induced hepatitis. In a case-control study, 60 consecutive patients with evidence of ATT-induced hepatitis were studied to assess the possible association of the above-mentioned factors with ATT-induced hepatitis. Body mass index was found to be significantly lower in ATT-induced hepatitis patients $(17.2 \pm 2.7)$ than in controls $(19.5 \pm 3.3)$ $(p<0.05)$. Pyrazinamide was used in addition to isoniazid and rifampicin in a significantly higher percentage of patients in the ATT-induced hepatitis group (70\%) as compared with those in the control group (42\%). No significant differences were observed between the two groups with regard to the rest of the parameters.
\end{abstract}

Keywords: antituberculosis treatment, hepatotoxicity, malnutrition

\section{Introduction}

Tuberculosis is becoming an increasingly important problem worldwide, especially with the alarming increase in the incidence of acquired immunodeficency syndrome (AIDS). ${ }^{1}$ Drug-induced hepatotoxicity is a

All India Institute of Medical Sciences, New Delhi-110029, India

Department of

Gastroenterology

J Singh

A Arora

PK Garg

VS Thakur

RK Tandon

Department of

Medicine

JN Pande

Correspondence to

Prof RK Tandon

Accepted 15 February 1995 the considerable controversy, the present study was aimed at clarifying the role of the above factors as predictive markers for the development of ATT-induced hepatitis.

\section{Patients and methods}

In this case-control study, 60 consecutive patients with ATT-induced hepatitis attending the out-patients department or admitted to the All India Institute of Medical Sciences, New Delhi, formed the study group. The criteria followed for diagnosing hepatitis were clinical manifestations of hepatitis along with serum aminotransferase levels more than twice the normal upper limit. An equal number of consecutive patients with tuberculosis who received the full course of ATT without developing hepatitis formed the control group. These patients were started on ATT in our hospital and were followed up regularly while they were receiving ATT. In all patients who presented to us with acute hepatitis while on ATT, sera were analysed for the presence of markers of acute viral hepatitis A, B and C (IgM anti-HAV, IgM anti-HBc and anti-HCV antibodies by ELISA, respectively). We excluded those patients whose results of serologic tests indicated that the acute hepatitis was of viral origin. All patients underwent a detailed clinical assessment. The details of the ATT received including the nature of drugs, dosage and duration, patient compliance and intake of other potentially hepatotoxic agents including alcohol were carefully recorded. A daily consumption of more than $40 \mathrm{~g}$ of alcohol for at least five years was considered as chronic alcoholism. The nutritional status of the patients was estimated by calculating the body mass index (BMI) (weight in kg/height in $\mathrm{m}^{2}$ ). Malnutrition was considered to be present if BMI was less than $18.5 .^{14}$ The presence of chronic liver disease was established by liver function tests, endoscopy, ultrasonography and liver biopsy (wherever possible).

A complete liver function profile including serum bilirubin, serum aminotransferases, total protein and serum albumin, serum alkaline phosphatase and hepatitis $B$ virus surface antigen was carried out in all patients of both groups. Determination of the acetylator phenotype was done by the serum sulfadimidine test. ${ }^{15}$ Antituberculosis drugs were discontinued for at least $\mathbf{4 8}$ hours before this test was carried out. Acetylator status was determined after the resolution of hepatitis in studies. However, contradictory results have been reported by other workers ${ }^{11-13}$ and consensus regarding their role is lacking. In view of 
patients with ATT-induced hepatitis. Biochemical tests of hepatocellular injury were repeated every week till recovery in patients with ATT-induced hepatitis.

Statistical analysis was done by applying Student's $t$-test for continuous variables and chi-square test with Yates' correction for dichotomous variables. A p value of less than 0.05 was regarded as significant.

\section{Results}

There were 60 patients each in the study and control groups. The mean age of the patients in the ATT-induced hepatitis group was $40.7 \pm 18$ years and the majority of the cases $(69 \%)$ were aged between $14-50$ years. The mean ages of the patients in the study and control groups were not significantly different (table 1). ATT-induced hepatitis was seen almost equally in males and females (table 1).

The interval between the start of ATT and the appearance of hepatotoxicity varied from three to 135 days with a mean of $29.1 \pm 27.6$ days. In two-thirds of cases, hepatitis was evident within the first month of starting ATT while in nine cases $(15 \%)$ the onset was delayed for more than two months after the start of treatment.

There was no significant difference between the two groups regarding the number of patients with a history of chronic alcoholism, chronic liver disease and hepatitis $\mathbf{B}$ virus carrier state. Determination of the acetylator status was possible in 32 patients in the study group and 54 patients in the control group. The percentages of rapid and slow acetylators were similar in the two groups (table 1).

BMI was significantly lower in the ATTrelated hepatitis patients compared with those in the control group (table 1). In patients with ATT-induced hepatitis, evidence of malnutrition (BMI < 18.5) was found in 32 patients $(53.2 \%)$. Patients with malnutrition received significantly higher doses of isoniazid, rifampicin and pyrazinamide compared with patients without malnutrition (table 2).

All the patients in the study and control groups had received isoniazid and rifampicin. However, in addition, pyrazinamide was used in a significantly higher percentage of patients in the ATT-induced hepatitis group (70\%) compared with patients in the control group $(38.2 \%)$

\section{Discussion}

There is wide disparity in the reported incidence of ATT-induced hepatitis in different studies, the incidence being much higher in studies from India $(8-39 \%))^{3,16,17}$ than in those from Western countries $(2-3 \%) .^{18-20}$ Why only some patients who receive ATT develop hepatitis is not clear. Whether some host factors, genetic predisposition, environmental factors, or some interaction among various factors is responsible is not known. The reported mortality from ATTinduced hepatitis after the development of jaundice varies from $4-12 \% \cdot{ }^{21,22}$ No consensus
Table 1 Characteristics of ATT-induced hepatitis

\begin{tabular}{|c|c|c|}
\hline & $\begin{array}{l}\text { Patients } \\
(n=60)\end{array}$ & $\begin{array}{l}\text { Controls } \\
(n=60)\end{array}$ \\
\hline Age (years) & $40.76 \pm 18.02$ & $37.52 \pm 13.39$ \\
\hline Sex M:F & $33: 27$ & $34: 26$ \\
\hline Chronic alcoholism & $2(3.33 \%)$ & $5(8.33 \%)$ \\
\hline Chronic liver disease & $4(6.67 \%)$ & $5(8.33 \%)$ \\
\hline HBV carrier & none & $3(5 \%)$ \\
\hline $\begin{array}{l}\text { Acetylator status } \\
\text { (rapid:slow) }\end{array}$ & $22: 10$ & $35: 19$ \\
\hline $\begin{array}{l}\text { BMI ratio } \\
\quad(\text { mean } \pm S D)\end{array}$ & $17.22 \pm 2.75$ & $19.50 \pm 3.33^{\star}$ \\
\hline $\begin{array}{c}\text { Treatment with } \\
\text { pyrazinamide }\end{array}$ & $42(70 \%)$ & $23(38.26 \%)^{\star}$ \\
\hline
\end{tabular}

${ }^{*} \mathrm{p}<0.05 ; \mathrm{HBV}$ : hepatitis B virus; BMI : body mass index.

Table 2 Comparison of daily drug dosages ( $\mathrm{mg} / \mathrm{kg}$ body weight) between patients with and without malnutrition (mean $\pm S D$ )

\begin{tabular}{lcc}
\hline Drug & $\begin{array}{l}\text { No malnutrition } \\
(n=32)\end{array}$ & $\begin{array}{l}\text { Malnutrition } \\
(n=28)\end{array}$ \\
\hline Isoniazid & $6.01 \pm 0.79$ & $6.81 \pm 0.67^{\star}$ \\
Rifampicin & $9.00 \pm 1.17$ & $10.31 \pm 1.27^{\star}$ \\
Pyrazinamide & $27.50 \pm 3.08$ & $32.14 \pm 4.14^{\star}$ \\
\hline
\end{tabular}

${ }^{\star} \mathrm{p}<0.001$.

exists regarding any predictive factors which can reliably identify those patients who form a high risk group for the development of hepatitis.

In earlier studies it has been observed that the risk of ATT-induced hepatitis increases with advancing age, the highest incidence being in individuals older than 50 years. ${ }^{5}$ In the present study, no significant correlation of age with ATT-induced hepatitis was found. Although it has been reported that women are more prone to develop ATT-induced hepatitis, ${ }^{6}$ we did not find any female preponderance in our study patients. Such a lack of sex difference has been reported earlier. ${ }^{11}$

Contrary to observations in earlier studies, ${ }^{6,7}$ no significant difference was found in the prevalence of chronic alcoholism or chronic liver disease among patients in the study and control groups. This discrepancy is perhaps due to the small number of alcoholics in our study population (two) and also due to the fact that almost half of our patients were women, who do not traditionally consume alcohol in India. Another study has also reported that the presence of chronic liver disease does not confer any additional risk of ATT-induced hepatitis. ${ }^{12}$

There is considerable controversy regarding the relationship of acetylator status to ATTinduced hepatotoxicity. Both rapid ${ }^{9,23}$ and slow acetylators ${ }^{3,24}$ have been reported to be more susceptible to drug-induced hepatitis. In our study the percentages of rapid and slow acetylators were similar in the ATT-related hepatitis patients and in the control group. Similar observations have been made by Gur- 
murthy et al from a large study in South Indian patients ${ }^{13}$ and by our group in a smaller number of patients. ${ }^{25}$

A recent study from Taiwan reported a higher incidence of ATT-induced fulminant and subacute hepatic failure in hepatitis $B$ virus carriers compared to noncarriers. ${ }^{8}$ In our study no significant difference in the number of hepatitis B virus carriers was observed in the study and control groups; these results are in agreement with those of another study which found no increased risk in hepatitis $B$ virus carriers. ${ }^{26}$

Low nutritional status is considered to be one of the factors contributing to the relatively high incidence of ATT-related hepatitis in studies from the developing countries. ${ }^{10,27}$ Drug metabolism pathways including acetylation pathways have been shown to be deranged in states of protein energy malnutrition. ${ }^{28}$ In the present study, the BMI of patients with ATT-induced hepatitis was significantly lower than that of patients in the control group. Patients with malnutrition who developed drug-induced hepatitis also had a tendency to receive comparatively higher doses of antituberculosis drugs (table 2).

In our study, pyrazinamide was used, in addition to isoniazid and rifampicin, in a significantly higher proportion of ATTinduced hepatitis patients compared to patients in the control group. This contradicts recent reports that pyrazinamide is not hepatotoxic when used at low doses. ${ }^{3,29}$ In earlier reports of pyrazinamide hepatotoxicity, the drug was used in higher dosages. ${ }^{30}$ Patients with severe forms of tuberculosis are known to be at a higher risk of developing ATT-induced hepatitis. ${ }^{3,31}$ Since a large number of patients in our series had been started on ATT by physicians outside this hospital and reported to us only after the development of drug-induced hepatitis, we could not estimate reliably the pretreatment severity of their underlying tuberculosis. This may have confounded our observation regarding the use of pyrazinamide which we believe requires validation in a larger number of patients.

We conclude that lower nutritional status is the only factor studied that is definitely

1 Davidson PT, Quoc Le H. Drug treatment of tuberculosis 1992. Drugs 1992; 43: 651-73.

2 Gangadharam PRJ. Isoniazid, rifampin and hepatotoxicity. Am Rev Respir Dis 1986; 133: 963-5.

3 Parthasarathy R, Raghupati Sarma G, Janardhanam B, et al. Parthasarathy R, Raghupati Sarma G, Janardhanam B, et al. of tuberculosis with short course regimens containing of tuberculosis with short course regimens containing
isonazid, rifampicin and pyrazinamide. Tubercle 1986; 67: isonazid,

4 Mahashur AA, Prabhudesai PP. Hepatitis and antitubercular therapy. F Assoc Physicians India 1991; 39: 595-6.

5 US Centers for Disease Control. National consensus conference on tuberculosis. Preventive treatment of tuberculosis. Chest 1985; 87 (suppl 2): 128-32.

6 Gronhagen-Riska C, Hellstrom PE, Froseth B. Predisposing factors in hepatitis induced by isoniazid-rifampin treatmen of tuberculosis. Am Rev Respir Dis 1978; 118: 461-6.

7 Riska N. Hepatitis cases in isoniazid groups and in a control group. Bull Int Union Tuberc 1976; 51: 203-6.

8 Wu JC, Lee SD, Yeh PF et al. Isoniazid-rifampin induced hepatitis in hepatitis B carriers. Gastroenterology 1990; 98: 502-4.

9 Mitchell JR, Thorgeirsson UP, Black M, et al. Increased incidence of isoniazid hepatitis in rapid acetylators: possible relation to hydrazine metabolites. Clin Pharmacol Ther 1975; 18: $70-9$.
Side-effects of common antituberculosis drugs

Isoniazid: hypersensitivity reaction, rash, hepatitis, peripheral neuropathy, lupus-like syndrome, psychosis

Rifampicin: flu-like syndrome, hepatotoxicity, rash, hypersensitivity, drug interaction, immunosuppression

Pyrazinamide: hepatotoxicity, increased uric acid levels, arthritis, gastrointestinal symptoms

Ethambutol: $\quad$ optic neuritis, mental confusion, joint pains

Streptomycin: ototoxicity, nephrotoxicity, vestibular dysfunction

Cycloserine: CNS side-effects such as headache, tremors, confusion, psychosis, depression

\section{Summary points}

- tuberculosis, a disease common in third world countries, is making a resurgence in developed countries with the prevalence of HIV infection

- ATT-induced hepatitis is quite common

- various factors which might predispose to the development of ATT-induced hepatitis have been suggested (acetylator status, underlying chronic liver disease, hepatitis B carrier status, alcoholism, malnutrition, etc.)

- the present study has shown that malnutrition is a predisposing factor for the development of ATT-induced hepatotoxicity, while age, sex, alcohol intake, chronic liver disease and acetylator status are not

- pyrazinamide, in combination with isoniazid and rifampicin, appears to be associated with an increased incidence of hepatotoxicity

associated with the development of ATTinduced hepatitis.

The authors thank Hind Chemicals Ltd, Kanpur, for having given them a grant which helped in analysing the viral markers in study patients.

10 Rugmini PS, Mehta S. Hepatotoxicity of isoniazid and rifampin in children. Indian Pediatr 1984; 21: 119-26.

11 Taneja DP, Kaur D. Study on hepatotoxicity and other side effects of antituberculosis drugs. F Indian Med Assoc 1990; 88: $278-80$.

12 Girling DJ. The hepatic toxicity of antituberculosis regimens containing isoniazid, rifampicin, and pyrazinamide. Tubercle 1978; 59: 13-32.

13 Gurumurthy $\mathrm{P}$, Krishnamurthy MS, Nazareth O, et al. Lack of relationship between hepatic toxicity and acetylator phenotype in three thousand South Indian patients during treatment with isoniazid for tuberculosis. Am Rev Respir Dis 1984; 129: 58-61.

14 National Institute of Nutrition. BMI and mortality rate-a 10 year prospective study. National Institute of Nutrition Annual Report, 1989, pp 1-13.

15 Rao KVN, Mitchison DA, Nair NGK, Prema K, Tripathi SP. Sulphadimidine acetylation test for classification of patients as slow or rapid inactivators of isoniazid. $B M \mathcal{F} 1970$; 3: 495-7.

16 Ramchandran P. Chemotherapy of tuberculous meningitis with isoniazid plus rifampin: interim findings in a trial in whildren. Indian $\mathcal{f}$ Tuberc 1980; 27: 54-7.

17 Rao NK, Wadia RS, Karve SR, Grant KB. Hepatotoxicity of antituberculous drugs. $\mathcal{F}$ Assoc Physicians India 1982; 30:
295-8. 
18 O'Brien RJ, Long MW, Floy Cross FS, Lyle MA, Snider $\mathrm{DE}, \mathrm{Jr}$. Hepatotoxicity from isoniazid plus rifampin among children treated for tuberculosis. Pediatrics 1983; 72: 491-9.

19 Dutt AS, Moers D, Stead WW. Short-course chemotherapy for tuberculosis with mainly twice weekly isoniazid and rifampin. Community physicians seven year experie
mainly outpatients. Am $\mathscr{\text { I Med }} 1984 ; 777: 233-42$.

20 Steele MA, Burk RF, DesPrez RM. Toxic hepatitis with isoniazid and rifampicin - a meta-analysis, Chest 1991; 99: 465-71.

21 Kopanoff DE, Snider JE, Jr, Caras GJ. Isoniazid related hepatitis. A US public health service cooperative surveillance study. Am Rev Respir Dis 1978; 117: 991-1001.

22 Black M, Mitchell JR, Zimmerman HJ, Ishak KG, Epler GR. Isoniazid associated hepatitis in 114 patients. Gastroenterology 1975; 69: 289-302.

23 Ellard GA. A slow release preparation of isoniazid: pharmacological aspects. Bull Int Union Tuberc Lung Dis 1976; macological

24 Lal S, Singhal SN, Burley DM, Crossley G. Effect of rifampicin and isoniazid on liver function. $B M \mathcal{F}$ 1972; 1: 148-50.
25 Singh J, Garg PK, Thakur VS, Tandon RK. Antitubercular treatment induced hepatotoxicity: does acetylator status matter? Indian f Physiol Pharmacol 1995; 35: 43-6.

26 Mcglynn KA, Lustbader ED, Sharrar RG, Murphy EC, London WT. Isoniazid prophylaxis in hepatitis B carrier. Am Rev Respir Dis 1986; 134: 666-8.

27 Ansari MM, Beg MH, Haleem S. Hepatitis in patients with surgical complications of pulmonary tuberculosis. Indian $f$ Chest Dis Allied Sci 1991; 33: 133-8.

28 Buchanan N, Eyberg C, David MD. Isoniazid pharmacokinetics in kwashiorker. S Afr Med $\mathcal{F} 1979$; 56: 299-300.

29 British Thoracic Society. A controlled trial of 6 month chemotherapy in pulmonary tuberculosis: results during the 36 months after the end of chemotherapy and beyond. $\mathrm{Br} \mathcal{F}$ Dis Chest 1984; 78: 330-6.

30 Macleod HM, Hay D, Steward SM. The use of pyrazinamide plus isoniazid in the treatment of pulmonary

31 Kumar A, Misra PK, Mehotra R, Govil YC, Rana GS. Hepatotoxicity of rifampin and isoniazid. Is it all dru induced hepatitis? Am Rev Respir Dis 1991; 143: 1350-2. 